\title{
Pharmacokinetic Analysis of the Monoclonal Antibody A7-Neocarzinostatin Conjugate Administered to Nude Mice
}

\author{
Kazuya Kitamura, Toshio Takahashi, Akinori \\ Noguchi, Hiroshi Tsurumi, Ken-ichiroh Takashina, \\ Junichi Okuzumi and Toshinaru Yamaguchi \\ The First Department of Surgery, Kyoto Prefectural \\ University of Medicine, Kyoto 602
}

\begin{abstract}
Kitamura, K., Takahashi, T., Noguchi, A., Tsurumi, H., Takashina, K., Окиzumi, J. and Yamaguchi, T. Pharmacokinetic Analysis of the Monoclonal Antibody A7-Neocarzinostatin Conjugate Administered to Nude Mice. Tohoku J. Exp. Med., 1991, 164 (3) 203-211 — The pharmacokinetics of a disulfide linked conjugate of a murine monoclonal antibody A7 with neocarzinostatin (A7-NCS) was studied following its intravenous administration to nude mice. Disappearance of the conjugate from the circulation was biphasic: an early rapid phase was followed by a much slower phase. The conjugate was removed from the blood circulation with a half-life of $12 \mathrm{hr}$, showing nearly the same kinetics as the free antibody. Sodium dodecyl sulfate-polyacrylamide gel electrophoresis analysis showed that the disulfide linkage in A7-NCS was stable at least for $48 \mathrm{hr}$ after administration of the conjugate to nude mice. The conjugate concentration in a human colon cancer SW1116 derived tumor reached maximum at $24 \mathrm{hr}$ after injection and remained high for an additional $24 \mathrm{hr}$. The passive hemagglutination inhibition assay revealed that NCS in the conjugated form can be efficiently delivered to the target tissue. The present report indicates that A7-NCS was sufficiently stable in circulation to reach the target tumor without releasing NCS. A7-NCS ; stability ; tumor localization
\end{abstract}

A recent approach to cancer chemotherapy is the use of monoclonal antibodies as carriers of toxin or drugs. There are now several reports of the construction and clinical application of monoclonal antibody-drug conjugates (Masuho et al. 1982; Pimm et al. 1982; Thorpe and Ross. 1982 ; Kanellos et al. 1985), whereas there are few studies on the in vivo phamacokinetics of the conjugate. To achieve effective therapy in vivo, the immunoconjugate must remain in circulation at a sufficiently high level for a sufficiently long time to allow binding to tumor cells. Most commonly, an antibody is linked to an anti-cancer reagent

Received April 19, 1991; revision accepted for publication June 15, 1991.

Reprint requests to: Kazuya Kitamura, the First Department of Surgery, Kyoto Prefectural University of Medicine, 465 Kawaramachi-hirokohji, Kajii-cho, Kamikyo-ku, Kyoto 602, Japan. 
through a disulfide bond by the introduction of a specific thiol reacting group on to the immunoglobulin (Carlsson et al. 1978; Masuho et al. 1982 ; Thorpe an Ross 1982 ; Noguchi et al. 1991). However, it has been suggested that the exposed disulfide linkage may be rapidly reduced, causing poor localization of the conjugate in tumor tissue (Thorpe and Ross 1982; Trowbridge and Domingo 1982 ; Strand et al. 1984). Recently, we coupled the murine monoclonal antibody A7 by a disulfide linkage to the anti-cancer drug-neocarzinostatin (NCS) which has a molecular weight of 10,700 daltons, and showed that A7-NCS had a powerful tumoricidal effect both in nude mice and in patients. Although considerable attention has been paid to the relationship between injected immunoconjugates and their tumoricidal effect, little is known about the pharmacokinetics of the conjugate. The present study was undertaken to elucidate the in vivo pharmacokinetics of a conjugate, particularly with respect to the disulfide linkage connecting the antibody with NCS.

\section{Materials and Methods}

Materials. The murine monoclonal antibody A7 (Mab A7), which is an $\operatorname{IgG}_{1}$, was developed as described by Kotanagi et al. (1986). Mab A7 has been shown to highly react with colon cancer and to recognize a $45 \mathrm{kD}$ glycoprotein on the cell membrane (Kitamura et al. 1989). $\quad \mathrm{Na}^{125}$ I, carrier free, was purchased from Amersham International Plc. (Amersham, U.K.). Mab A7 or A7-NCS were labeled with ${ }^{125} \mathrm{I}$ by chloramine $\mathrm{T}$ as described by Greenwood et al. (1963). The iodinated proteins had a specific activity of $25 \mu \mathrm{Ci} / \mu \mathrm{g}$. Greater than $99 \%$ of the radioactivity present in the radiolabeled materials was precipitated by $25 \%$ trichloroacetic acid in both Mab A7 and A7-NCS. Neocarzinostatin, an anti-cancer polypeptide with a molecular weight of 10,700 was provided by Pola Pharmaceutical Corp. (Yokohama). N-succinimidyl 3-(2-pyridyldithio) propionate (SPDP) and dithiothreitol (DTT) were purchased from Pharmacia Fine Chemicals (Uppsala, Sweden). The human colon cancer cell line-SW1116 was cultured in Roswell Park Memorial Institute medium 1640 (RPMI 1640) with $10 \%$ fetal calf serum.

Preparation of antibody-drug conjugate. Mab A7 was conjugated with NCS as described by Fukuda (1985). Briefly, NCS was incubated with a four-fold molar excess of SPDP in $0.1 \mathrm{M}$ phosphate buffer, $\mathrm{pH} 6.5$ at $25^{\circ} \mathrm{C}$ for $30 \mathrm{~min}$. The 3-(2-pyridyldithio)propionated (PDP) NCS was reduced with $10 \mathrm{mM}$ of DTT in $0.1 \mathrm{M}$ acetate buffer, $\mathrm{pH} 4.5$ at $25^{\circ} \mathrm{C}$ for $30 \mathrm{~min}$. The resulting thiol group-introduced NCS (SH-NCS) was passed immediately through a Sephadex G-25 column (Pharmacia Fine Chemical). Mab A7 was incubated with a ten-fold molar excess of SPDP in $0.1 \mathrm{M}$ phosphate buffer, $\mathrm{pH} 6.5$ at $25^{\circ} \mathrm{C}$ for 30 min. PDP was purified by gel filtration on a Sephadex G-25 column. Finally, PDP was mixed with a six-fold molar excess of SH-NCS and allowed to stand at $25^{\circ} \mathrm{C}$ overnight in the dark. The mixture was then applied to a Sephacryl S-200 column (Pharmacia Fine Chemical) equilibrated with PBS, pH 6.0 and eluted with the same buffer. The peak fractions were pooled, concentrated, sterilized, and stored at $-20^{\circ} \mathrm{C}$ in the dark. The conjugation ratio was 2 molecules of NCS per molecule of Mab A7. The molecular weight was determined to be 170,000 by sodium dodecyl sulfate-polyacryl amide gel electrophoresis (SDS-PAGE).

Blood clearance. Groups of four adult male nude mice (BALB/c, nu/nu) were given $i v$ injections of Mab A7 or A7-NCS containing $5 \times 10^{6} \mathrm{cpm}$ of ${ }^{125} \mathrm{I}$. Blood samples were drawn from the tail vein of the mice with heparinized capillaries at 5, 10, 15, 30, 60 and 120 $\min$ (initial phase). In the late phase, samples were taken at $0.25,0.5,1,2,6,12,24,48$ and 
$72 \mathrm{hr}$ after injection. The radioactivity of the blood sample was measured in a gamma counter.

Tumor localization of the conjugate. SW1116 was used in this study because A7-NCS is highly cytotoxic to the cell line and tumoricidal to SW1116 bearing nude mice (Takahashi et al. 1988). The SW1116 xenograft initiated from a tumor cell line was passed subcutaneously into nude mice. Mice developed a palpable tumor weighing between $0.1 \mathrm{~g}$ and $0.5 \mathrm{~g} 2$ weeks after injection. Groups of four nude mice were given $i v$ injection $5 \times 10^{6} \mathrm{cpm}$ of ${ }^{125} \mathrm{I}$ labeled A7-NCS. The mice were killed at 12, 24,48 and $72 \mathrm{hr}$ after injection, and the radioactivity in resected tumors was counted in a gamma counter. The data was expressed as a percentage of the injected dose per gram of tumor.

Determination of NCS concentration in target tumor. A7-NCS or NCS (150 U as NCS per mouse) were intravenously administered to each group of four tumor bearing nude mice which were sacrificed one day after injection. Tumors were removed, weighed, minced in PBS and then centrifuged. NCS concentration was determined by passive hemagglutination inhibition (PHI) (Mukojima and Kimura 1974).

$S D S-P A G E$ analysis. Stability of the disulfide linkage between Mab A7 and NCS was analyzed by SDS-PAGE (Laemmli 1971). ${ }^{125}$ I radiolabeled A7-NCS $\left(5 \times 10^{6} \mathrm{cpm}\right.$ ) was intravenously injected into nude mice. Blood samples were drawn from the tail vein with heparinized capillaries at various time intervals. The samples were centrifuged at 10,000 rpm and plasma was taken for gamma counting. Volumes of plasma samples containing $20,000 \mathrm{cpm}$ each were electrophoresed on SDS-PAGE. The gel was dried and autoradiographed using Kodak film with an exposure period of one day at $-70^{\circ} \mathrm{C}$.

In a separate experiment, radiolabeled A7-NCS solution $\left(2 \times 10^{5} \mathrm{cpm}\right)$ was incubated with $500 \mu 1$ human serum at $37^{\circ} \mathrm{C}$. Samples were then taken at various time intervals and stored at $-20^{\circ} \mathrm{C}$. Serum samples $(50 \mu \mathrm{l})$ containing $2 \times 10^{4} \mathrm{cpm}$ of radiolabeled A7-NCS were separated by SDS-PAGE followed by autoradiography. These experiments were repeated twice.

\section{Results}

Blood clearance. Blood concentrations of ${ }^{125} \mathrm{I}$ labeled A7-NCS following intravenous injection to nude mice were determined at various times after administration. In the initial phase, the concentration of A7-NCS was maintained at a maximum dose for an initial $30 \mathrm{~min}$ after injection (Fig. 1). Thus, results were expressed as a percentage of the concentration at 30 min after injection and plotted against time in a semi-logarithmic plot. The clearance curve was biphasic. An initial rapid phase was followed by a slower phase (Fig. 2). As shown in Fig. 2, the conjugate displayed a nearly identical kinetic profile to that of free antibody, with a $12 \mathrm{hr}$ half-life in the rapid phase and a $48 \mathrm{hr}$ half life in the slower phase. The blood concentration of A7-NCS was reduced to $36 \%$ at 24 $\mathrm{hr}, 24 \%$ at $48 \mathrm{hr}$ and $18 \%$ at $72 \mathrm{hr}$ after injection, respectively.

Tumor localization. Fig. 3 shows the tumor distribution of radiolabeled A7-NCS at various times after injection into tumor bearing nude mice. The percentage of the injected dose per gram of tumor for A7-NCS reached a maximum $24 \mathrm{hr}$ after injection and subsequently maintained the high level for a further 48 hr, with 5.8, 7.5, 7.2 and $5.5 \%$ for $12,24,48$ and $72 \mathrm{hr}$ after administration, respectively. Tumor : blood ratios were $0.55,0.88,1.05$ and 1.52 for $12,24,48$ and $72 \mathrm{hr}$ after administration, respectively. These findings indicate that most parts of the 


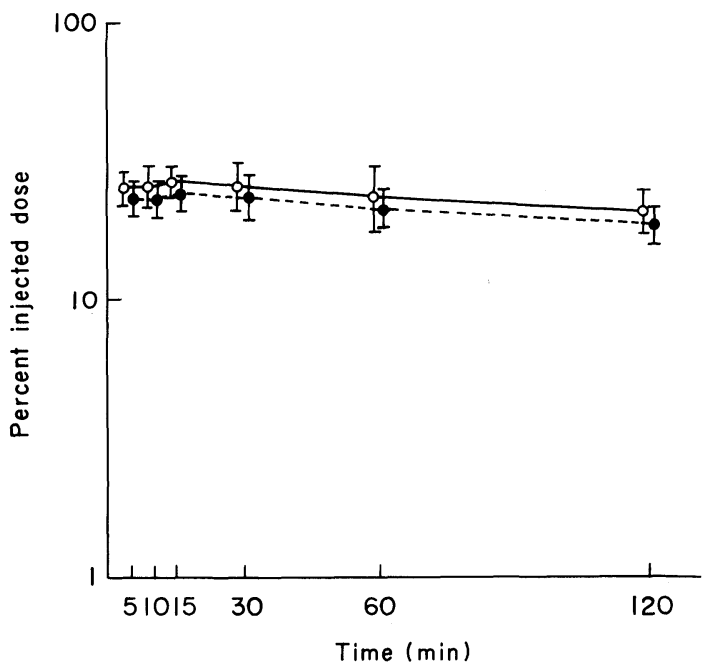

Fig. 1. Initial blood circulation. ${ }^{125}$ I-labeled Mab A7 or A7-NCS (5 $\left.\times 10^{6} \mathrm{cpm}\right)$ was injected intravenously into nude mice. Blood samples were taken at 5, 10, 15, 30, 60 and 120 min after injection for the initial phase. Samples were then gamma counted. Data are expressed as means \pm s.E. $(n=4)$. O, Mab A7 ; •, A7-NCS.

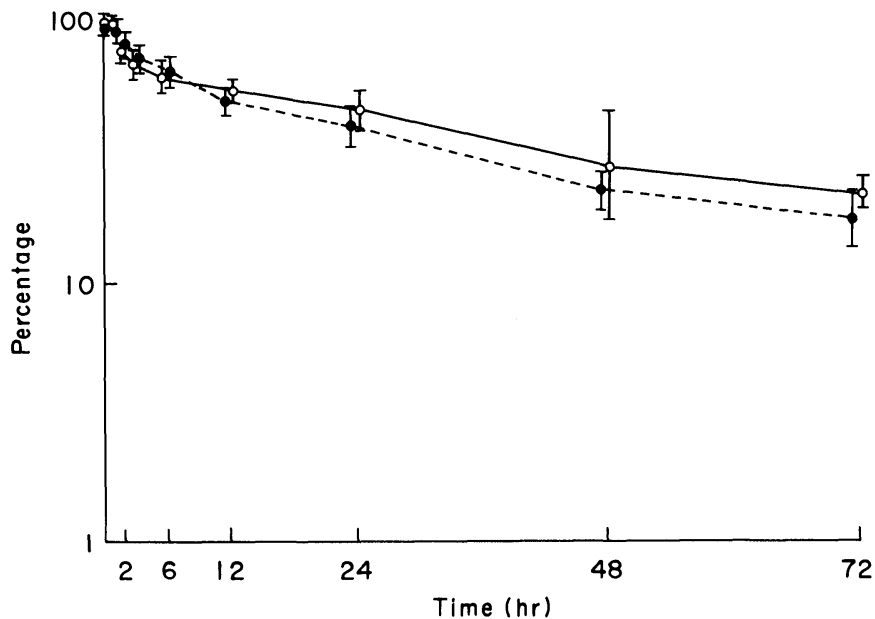

Fig. 2. Late blood circulation. ${ }^{125}$ I-labeled Mab A7 or A7-NCS $\left(5 \times 10^{6} \mathrm{cpm}\right)$ were injected intravenously into nude mice. Blood samples were taken at $0.25,0.5,1,2,6,12,24,48$ and $72 \mathrm{hr}$ after injection and gamma counted. The results are expressed as a percentage of the radioactivity remaining in the circulation with respect to the value at $30 \mathrm{~min}$ after injection and are plotted against time in a semi logarithmic plot. Data were mean \pm S.E. $(n=4) .0$, Mab A7; •, A7-NCS. 


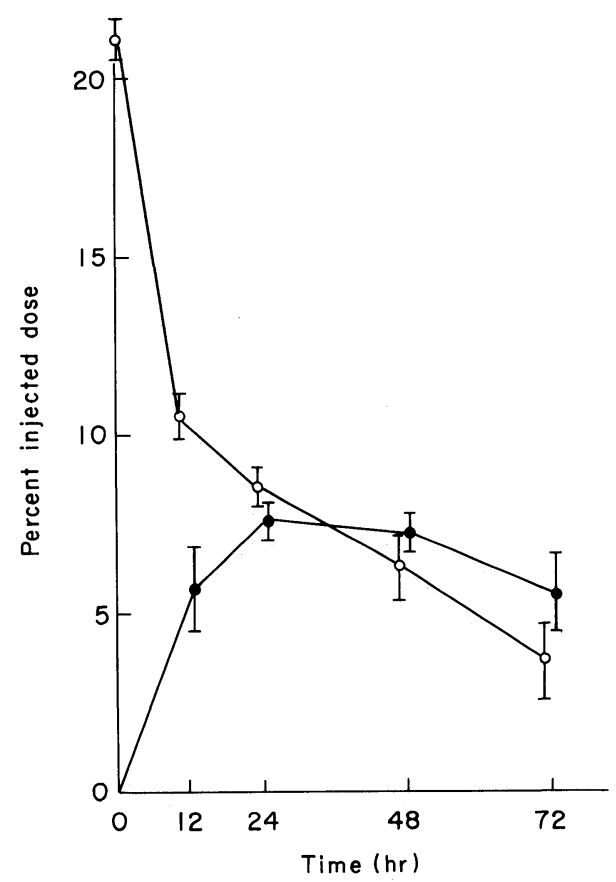

Fig. 3. Tumor and blood localization of A7-NCS. ${ }^{125}$ I-labeled A7-NCS was administered intravenously into tumor bearing nude mice. The mice were killed at various time intervals, then tumor and blood were removed for gamma-counting. Data are expressed as mean \pm s.e. $(n=4)$. $\bigcirc$, blood;

$\bullet$ tumor.

conjugate uptaken by the tumor was responsible for the initial $48 \mathrm{hr}$ accumulation after injection and thereafter were retained in the tumor.

NCS concentration in target tumors to which A\%-NCS or NCS was administered. To examine whether the NCS portion of the conjugate could reach the target tumor, the NCS concentration in the tumor was measured by PHI assay of tumor bearing nude mice. The PHI assay revealed that the NCS concentration was higher in the tumor administered with A7-NCS than that administered with free NCS (Fig. 4). The NCS concentration $24 \mathrm{hr}$ after injection was $0.6 \mathrm{U}$ for the A7-NCS administered group and $0.15 \mathrm{U}$ for the free NCS administered group, respectively.

SDS-PAGE analysis. The in vivo stability of A7-NCS was analyzed by SDS-PAGE followed by autoradiography. Plasma samples taken at different times after the injection were separated by SDS-PAGE. The injected conjugates contained two components (Mr. 170,000, Mr. 150,000), although they were too close to be seen in our system. After injection, the conjugate was broken down to give a long-lived product (Mr. 150,000) corresponding to the free antibody. The 


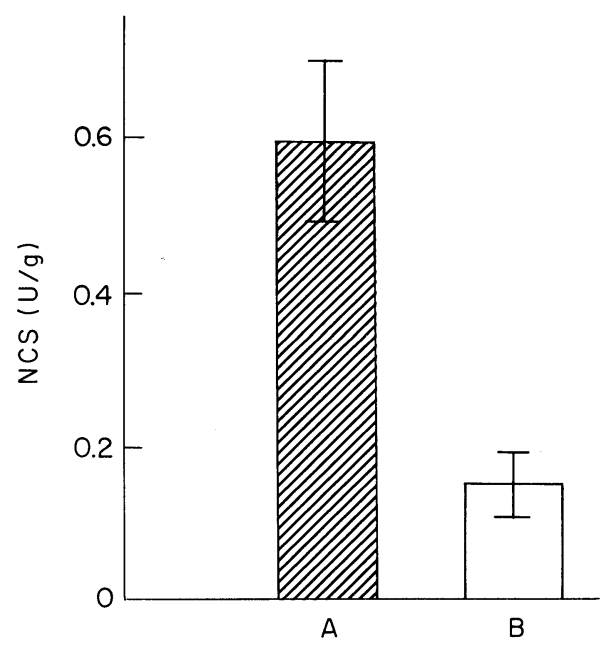

Fig. 4. NCS concentration in the tumor. A7-NCS or NCS (150 U per mouse) was administered intravenously into tumor bearing nude mice. Twenty four $\mathrm{hr}$ after administration, tumors were resected for NCS quantification by the passive hemagglutination inhibition assay. Data are expressed as units of NCS per gram of tumor (mean \pm s.e. $n=4)$. A, A7-NCS ; B, NCS.

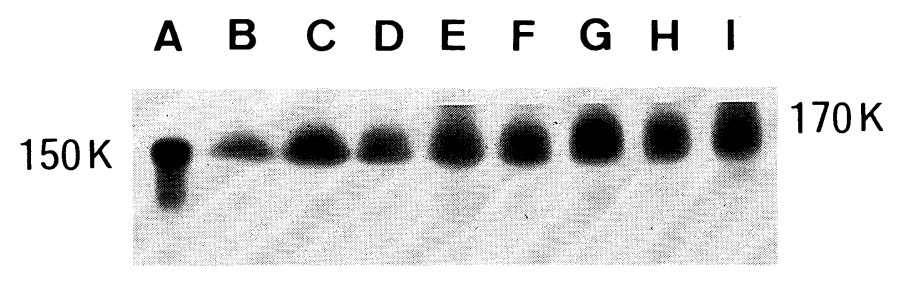

Fig. 5. SDS-PAGE of the conjugate administered to mice. ${ }^{125}$ I-labeled A7-NCS was administered intravenously into nude mice. Blood samples were taken at various time intervals and centrifuged at $3,000 \mathrm{rpm}$. Plasma samples containing $20,000 \mathrm{cpm}$ of proteins were separated by SDS-PAGE, then autoradiographed. Lane A, native Mab A7 ; Lanes B-H, plasma samples taken 96, 72, $48,24,12,6$ and $2 \mathrm{hr}$ after injection, respectively; Lane I, A7-NCS prior to injection.

conjugate released NCS $72 \mathrm{hr}$ after injection to give free antibody (Fig. 5). The conjugate incubated with human serum also contained two components for $48 \mathrm{hr}$ after initiation of incubation, and thereafter gave a single band (Fig. 6).

\section{Discussion}

There have been several reports discussing the breakdown of immunoconjugate in the blood stream (Carlsson et al. 1978; Trowbridge and Domingo 1982 ; Strand et al. 1984 ; Pimm et al. 1988). Some investigators have considered that a disulfide linkage of a monoclonal antibody with an anti-cancer reagent is inappropriate for targeting chemotherapy because of its instability (Thorpe and 


\section{A $\quad$ B $\quad$ C $\quad D \quad E$ $150 \mathrm{~K}=170 \mathrm{~K}$}

Fig. 6. SDS-PAGE of samples incubated with human serum. ${ }^{125}$ I-labeled A7NCS was incubated with human serum. Serum samples containing 20,000 cpm of radioactivity were taken at times indicated. Samples were treated in the same manner as described in Fig. 5. Lanes A-E, plasma samples taken at $96,72,48,24$, and $12 \mathrm{hr}$ of incubation, respectively.

Ross 1982 ; Trowbridge and Domingo 1982 ; Strand et al. 1984). A7-NCS has a strong tumoricidal effect in vivo (Fukuda 1985), though little is known about its pharmacokinetics. However, this conjugate has a disulfide linkage between Mab A7 and NCS, and is may be consequently reduced to release NCS before reaching the target tumor. The present study was undertaken to examine the tumor localization of the conjugate and the stability of the disulfide linkage.

To assess the stability of A7-NCS, ${ }^{125} \mathrm{I}$ labeled A7-NCS was intravenously administered to mice or incubated with human serum. Blood samples were then taken at various times for SDS-PAGE analysis followed by autoradiography. The results showed that A7-NCS retained sufficient NCS within $48 \mathrm{hr}$ after either administration to mice or initiation of incubation with human serum. In addition, we elucidated that NCS reached the target tumor when A7-NCS was given to tumor bearing nude mice. Masuda (1985) has also showed that NCS reached the target tumor at a sufficiently high level to allow uptake when A7-NCS was intravenously administered to tumor bearing nude mice. In spite of the use of SPDP as the linker, A7-NCS appears to have high stability with respect to the disulfide linkage, as compared with those of an antibody-ricin conjugate described in previous papers (Worrell et al. 1986 ; Blakey et al. 1987 ; Thorpe et al. 1987). This favorable property of A7-NCS could be attributed to its steric difference from other immunotoxin conjugates.

A7-NCS in blood circulation decreased with a profile identical to, although not precisely paralleling, that of the free antibody. In many cases, when an antibody is modified with a macromolecule such as ricin $\mathrm{A}$, the conjugate is cleared from the blood faster than the parent antibody. NCS is a macromolecular anti-cancer protein with a molecular weight of 10,700. Nevertheless, A7-NCS assumed an identical clearance pattern to that of free antibody and was reduced in the blood circulation with a half life of $12 \mathrm{hr}$ after injection. In a previous report, Fujita et al. (1979) have demonstrated that free NCS was cleared from blood circulation with a half life of several minutes. This implies that the blood half life of NCS was prolonged approximately 200-fold when conjugated with Mab A7. This prolongation would be beneficial and essential for cancer therapy. 
A7-NCS reached the tumor with a maximum at $24 \mathrm{hr}$ after administration and retained a high concentration for a further $48 \mathrm{hr}$. On the other hand, the blood concentration of A7-NCS was reduced in a time-dependent fashion to $36 \%$ and $24 \%$ of the maximum dose, $24 \mathrm{hr}$ and $48 \mathrm{hr}$ after injection, respectively. These different kinetics between blood and tumor concentrations of A7-NCS may cause its tumor retention after extravasation from blood vessels, partly as a result of endocytosis (Kitamura et al. 1990). As the conjugate concentration in the tumor reached a level higher than that of the blood at $48 \mathrm{hr}$ after injection, most of the tumor uptake would be dependent on the initial $24 \mathrm{hr}$ accumulation after injection. Thus, A7-NCS can carry sufficient NCS to a target tumor to achieve in vivo cytocidal effects, even though A7-NCS releases NCS $48 \mathrm{hr}$ after injection. This would also lead to the hypothesis in tumor targeting that an anti-cancer agent could fully reach the target tumor with a monoclonal antibody when the linkage of the two molecules was kept stable during the initial period after injection.

In conclusion, A7-NCS retained high stability in binding to a tumor in vivo. The excellent effect of this conjugate in vivo may be partly due to its stability.

\section{References}

1) Blakey, D.C., Watson, G.J., Knowles, P.P. \& Thorpe, P.E. (1987) Effect of chemical deglycosilation of ricin A chain on the in vivo fate and cytotoxic activity of an immunotoxin composed of ricin A-chain and anti-Thyl.1 antibody. Cancer Res., 47, 947-952.

2) Carlsson, J., Dression, H. \& Axen, R. (1978) Protein thiolation and reversible protein-protein conjugation. N-succinimydil 3-(2-pyridyldithio) propionate, a new heterobifunctional reagent. Biochem. $J ., 173,723-737$.

3) Fujita, H., Nakayama, N., Sawabe, T. \& Kimura, K. (1979) In vivo distribution and inactivation of neocarzinostatin. Jpn. J. Anti., 23, 471-478.

4) Fukuda, T. (1985) Study of targeting chemotherapy against gastrointestinal cancer: Preparation of anticancer drug-monoclonal antibody conjugate and investigation about its biological activities. Akita J. Med., 12, 451-468.

5) Greenwood, F.C., Hunter, W.M. \& Glover, J.S. (1963) The preparation of 131-I labeled human growth hormone of high specific radioactivity. Biochem. J., 89, 114123.

6) Kanellos, J., Pietersz, G.A. \& Mckenzie, I.F.C. (1985) Studies of methotrexatemonoclonal antibody conjugate or immunotherapy. J. Natl. Cancer Inst., 75, 319332.

7) Kitamura, K., Takahashi, T., Yamaguchi, T., Yokota, T., Noguchi, A., Amagai, T. \& Imanishi, J. (1989) Immunochemical characterization of the antigen recognized by the murine monoclonal antibody $\mathrm{A} 7$ against human colorectal cancer. Tohoku $J$. Exp. Med., 157, 83-93.

8) Kitamura, K., Takahashi, T., Noguchi, A., Takashina, K. \& Yamaguchi, T. (1990) Binding, internalization and the cytotoxicity of the monoclonal antibody A7neocarzinostatin conjugate into target cells. Tohoku J. Exp. Med., 161, 621-630.

9) Kotanagi, H., Takahashi, T., Masuko, T., Hashimoto, Y. \& Koyama, K. (1986) A monoclonal antibody against human colon cancers. Tohoku J. Exp. Med., 148, 353360 .

10) Laemmli, U.K. (1971) Cleavage of structural protein during the assembly of the head of bacteriophage T4. Nature, 227, 680-685. 
11) Masuho, Y., Kishida, K., Saito, M., Umemoto, N. \& Hara, T. (1982) Importance of the antigen-binding valency and the nature of the cross-linking bond in ricin A chain conjugate with antibody $J$. Biochem., 91, 1583-1591.

12) Masuda, T. (1985) In vivo distribution of neocarzinostatin conjugated with antihuman colon cancer-monoclonal antibody. Akita J. Med., 12, 469-480.

13) Mukojima, T. \& Kimura, K. (1974) Immunological assay of neocarzinostatin. Jap. J. Cancer Clin., 20, 245-248 (in Japanese)

14) Noguchi, A., Takahashi, T., Yamaguchi, T., Kitamura, K., Yokota, T., Takakura, Y., Hashida, M. \& Sezaki, H. (1991) Preparation and properties of the immunoconjugate composed of anti-human colon cancer monoclonal antibody and mitomycin C-Dextran conjugate. Bioconjugate., in press.

15) Pimm, M.V., Jones, J.A., Price, M.R., Middle, J.G., Embleton, M.J. \& Baldwin, R.W. (1982) Tumor localization of monoclonal antibody against a rat mammary carcinoma and suppression of tumor growth with adriamycin-antibody conjugate. Cancer Immunol. Immunother., 12, 125-134.

16) Pimm, M.V., Clegg, J.A., Garnett, M.C. \& Baldwin, R.W. (1988) Biodistribution and tumor localization of a methotrexate-monoclonal antibody $791 \mathrm{~T} / 36$ conjugate in nude mice with human tumor xenografts. Int. J. cancer, 41, 886-891.

17) Strand, M., Scheinberg, D.A. \& Gansom, O.A. (1984) Monoclonal antibody conjugate for tumor imaging and therapy. In: Cell Fusion: Gene Transfer and Transformation. Miles International Symposium 14, edited by R.F. Beers \& E.G. Basset, Raven Press, New York, pp. 385-395.

18) Takahashi, T., Yamaguchi, T., Kitamura, K., Suzuyama, H., Honda, M., Yokota, T., Kotanagi, H., Takahashi, M. \& Hashimoto, Y. (1988) Clinical application of monoclonal antibody-drug conjugates for immunotargeting chemotherapy of colorectal carcinoma. Cancer, 61, 881-888.

19) Thorpe, P.E. \& Ross, W.C.J. (1982) The preparation and cytotoxic properties of antibody-toxin conjugates. Immunol. Rev., 62, 119-158.

20) Thorpe, P.E., Wallace, P.M., Knowles, P.P., Relf, M.G., Brown, A.N.F., Watson, G.J., Knyba, R.E., Wawrzynczak, E.J. \& Blakey, D.C. (1987) New coupling agents for the synthesis of immunotoxins containing a hindered disulfide bond with improved stability in vivo. Cancer Res., 47, 5924-5931.

21) Trowbridge, I.S. \& Domingo, D.L. (1982) Prospects for the clinical use of cytotoxic monoclonal antibody conjugate in the treatment of cancer. Cancer Survey., 1, 542546.

22) Worrell, N.R., Cumber, A.J., Parnell, G.D., Mirza, A., Forrester, J.A. \& Ross, W.C.J. (1986) Effect of linkage variation on pharmacokinetics of ricin A chain-antibody conjugates in normal rats. Anti cancer Drug Des., 1, 179-188. 